\title{
EINIGE BEZEICHNUNGEN
}

Elektrische Feldstärke und elektrische Induktion: $\boldsymbol{E}$ und $\boldsymbol{D}$.

Feldatärke und Induktion des Magnetfeldes: $\boldsymbol{H}$ und $\boldsymbol{B}$.

Äußere elektrische und magnetische Feldstärken: $\mathfrak{E}, \mathfrak{H}$; Beträge $\mathfrak{E}, \mathfrak{F}$.

Dielektrische Polarisation: $\boldsymbol{P}$.

Magnetisierung: $\boldsymbol{M}$.

Integrales elektrisches und magnetisches Moment eines Körpers:

$\mathscr{P}$ und $\mathscr{M}$;

Beträge $\mathscr{P}$ und $\mathscr{M}$.

Dielektrische Permeabilität: $\varepsilon$.

Dielektrische Suszeptibilität: $\chi$.

Magnetische Permeabilität: $\mu$.

Magnetische Suszeptibilität: $\chi$.

Elektrische Stromdichte: $\boldsymbol{j}$.

Elektrische Leitfähigkeit: $\sigma$.

Absolute Temperatur: $T$.

Druck: $P$.

Volumen: $V$.

Dichten thermodynamischer Größen:

Entropiedichte $S$,

Dichte der inneren Energie $U$,

Dichte der freien Energie $F$,

Dichte des thermodynamischen Potentials $\Phi$.

Die gleichen Größen für den Gesamtkörper: $\mathscr{S}, \mathcal{U}, \mathcal{F}, \boldsymbol{g}$.

Chemisches Potential: $\zeta$.

Ein komplexer, zeitlich periodischer Faktor wird immer in der Gestalt $\mathrm{e}^{-i \omega t}$ geschrieben.

Volumenelement: $\mathrm{d} V$ oder $\mathrm{d}^{3} x ;$ Flächenelement: $\mathrm{d} \boldsymbol{f}$.

Stets benutzt wird die Summationskonvention für zwei wiederholte vektorielle und tensorielle dreidimensionale (lateinische Buchstaben) und zweidimensionale (griechische Buchstaben) Indizes.

Die Zeichen $\approx$, bzw. $\propto$ bedeuten:

$\approx$ angenähert gleich

$\sim$ größenordnungsmä ßig gleich

$\propto$ proportional 
Die anderen Bände dieser Lehrbuchreihe werden wie folgt zitiert

$$
\text { I - Band I ,Mechanik“ }
$$

II - Band II „Klassische Feldtheorie“

III - Band III ,Quantenmechanik“

IV - Band IV ,Relativistische Quantentheorie“

V - Band V ,Statistische Physik, Teil 1“

VI - Band VI ,Hydrodynamik“"

VII - Band VII ,Elastizitätstheorie“

IX - Band IX ,Statistische Physik, Teil 2“

X - Band X ,Physikalische Kinetik“" 